\title{
Can Children Take Advantage of Nao Gaze-Based Hints During GamePlay?
}

\begin{abstract}
This paper presents a study that analyzes the effects of robots' gaze hints on children's performance in a cardmatching game. We conducted a within-subjects study, in which children played a card game "Memory" in the presence of a robot tutor in two sessions. In one session, the robot gave hints to help the child find matching cards by looking at the correct match and, in the other session, the robot only looked at the child and did not give them any help. Our findings show that the use of gaze hints (help condition) made the matching task significantly easier and that children used a significantly fewer number of tries than without help. This study provides guidelines on how to design interactive behaviors for robots taking the role of tutors to elicit help-seeking behavior in children.
\end{abstract}

\section{Author Keywords}

Human-robot interaction, intentions, nonverbal behavior, social robotics, child-robot interaction, gaze-based interactions; intentions; attentional cues; facial orientation.

\section{INTRODUCTION}

Robots continue to gain popularity in areas of educational training, rehabilitation and therapeutic programs for children $[6,21]$. The research in this domain has demonstrated the prospect for social robots to support children's interactions [8, 12]. For instance, robots can provide appropriate personalized interactions by adapting to the cognitive and affective needs of a child, can give repetitive instructions or feedback, which may be challenging for individual teachers in classrooms. Furthermore, robots have been shown to elicit interest, which makes them useful tools for learning [16].

Among nonverbal behaviors, gaze is a primary source of information that humans use to communicate their intentions, emotions, or their attention $[4,15,20]$. An essential communicative property of gaze, in particular, is its ability to direct attention to objects of interest [19, 23].

Paste the appropriate copyright/license statement here. ACM now supports three different publication options:

- ACM copyright: ACM holds the copyright on the work. This is the historical approach.

- License: The author(s) retain copyright, but ACM receives an exclusive publication license.

- Open Access: The author(s) wish to pay for the work to be open access. The additional fee must be paid to ACM.

This text field is large enough to hold the appropriate release statement assuming it is single-spaced in Times New Roman 8-point font. Please do not change or modify the size of this text box.

Each submission will be assigned a DOI string to be included here.
Much psychological research has demonstrated that people are very sensitive to the gaze behavior of other people. For instance, infants are capable of following the gaze of their parents and caregivers at a very young age [7, 10,24]. Such attention directing gaze behavior facilitates the formation of essential social cognition abilities such as joint and shared attention, which are the foundation of learning [14, $15,23,38]$. One very insightful approach to the dynamics of human-robot communication is the Theory of Mind (ToM). Most remarkably, both psychologists Astington \& Jenkins [5] and roboticists -and in general experts in AIDautenhahn [12]; Krämer et al. [22] draw on the theories of mutual understanding to explain how human and robot may acquire the ability to represent each other's mind.Thus, being able to understand and provide gaze cues is an important aspect in developmental robotics.

While a lot of gaze research has taken a human-centered approach to examine the ability of humans to read and perceive social cues from robot gaze [1, 11, 13, 26, 34], many questions remain unclear, particularly on how children perceive and respond to gaze cues, and whether they are able to attribute intentions to a robot's gaze cues during child-robot interaction. In this paper, we examine whether children read/notice gaze hints in humanoid robots; if so, whether they they are able to interpret these cues appropriately and, finally, whether and under which conditions these social cues impact their performance and their cognitions about the robot. The ultimate purpose is to gain understanding of how gaze hints could be implemented as an efficient help mechanism for a robotbased tutoring system.

To answer the abovementioned questions, we designed a board game task in which a child plays "Memory" (matching card game) in the presence of a robot tutor. The robot tutor knows the positions of all the cards on the table. The aim was to examine if gaze hints provided by the tutor robot influenced the performance of the child in terms of execution: the number of tries (tries to pick a card) and the overall time the participant takes to complete the game. We conducted a two-session repeated measure interaction; in one of the sessions, the robot tutor gave hints to help the child find the matching cards by looking at the correct match (help condition). In the other case, the robot tutor kept looking at the child and did not give any help. We analyzed the game sessions to measure performance (time and number of attempts) of Help vs. No_Help. 
Based on the the non-verbal theories of gaze [19, 20], we hypothesize that gaze hints will direct the attention of the children, either implicitly or explicitly to the matching card and, thus, children will perform better with the help of the robot tutor than without help.

In the following sections, we describe related work on gaze behavior in social human-robot interactions. We further describe the methodology and the design of child - robot experimental setup and outline the results of the study. Finally, we discuss the findings and limitations of our work and give directions for future work.

\section{RELATED WORK}

A lot of gaze research in human-robot interaction has taken a human-centered approach to examine the ability of humans to read, perceive, and interpret social cues exhibited by robot gaze. For example, in [27], authors consider the extent to which people can understand and attribute meaning to leakage signals using Geminoid and Robovie platforms. The authors claim that, in general, the gaze cue led to better performance and even better with Robovie than with Geminoid. A study by Admoni et al.[1] examined the features that make a robot appear to be attending to someone; their findings reveal that people recognize shorter, frequent fixations from a robot than longer, less frequent cues. Authors in [28] examined how people perceive gaze cues and head angles directed towards different target positions on a table when a human and NAO robot are sitting against each other as in board game scenarios. Findings show that when the head pitch angle is higher (24 \pm 2$)$ and the depth is less, approximately $20 \mathrm{~cm}$ from the robot, participants detect the positions with good accuracy.

Gaze has also been used to create more efficient interactions with robots or virtual agents [11, 34]. For example, Yoshikawa et al. [36] show that responsive robot gazes induce stronger feelings of being looked at compared to non-responsive gazes. Andrist et al. [3] combined facetracking and head detection functionalities to create conversational gazes for NAO platform. Evaluation of the gaze models showed they were perceived as more intentional. A lot of work has also addressed the role of gaze in physical human-robot interaction to support collaborative work and as a mechanism to control turntaking. For instance, in a dictation task, Palinko et al. [30] found that mutual gaze is an efficient means of controlling turn-taking with human partners with different needs. Yamazaki et al. [35] also demonstrated the importance of gaze timing in turn-taking interactions. Also, in a handover setting where the robot handed bottles to human subjects, [25] empirical evidence reveals that gaze cues can improve hand over timing and the subjective evaluation of the robot.

Several studies have explored how human behaviors differ in interactions with robots compared to humans. For instance, using a word-learning task [37], a micro-level analysis shows that participants spend more time gazing at the face of the robotic agent than at the human's face when naming objects. Also, in a comparative eye-tracking study [29], eye gaze metrics reveal that participants look more into the face of the robot compared to the human, particularly when seeking help to complete a cardmatching task. Boucher et al. [9] studied gaze effects in human-human and human-robot interaction on task completion. Their findings demonstrate that human participants can use the gaze cues of a human or of a robot partner in a physical interaction. The differences in how human behavior changes in interaction with humans compared to the robots has been attributed to many factors: for example, the novelty effect of the robot, [29,37], the robot's gaze being very overt with large head motions compared to more natural gaze movements that are much more subtle, as humans mostly use their eyes without much head movement.

In children robot interaction (CRI), a lot of research has focused on the use of robots in therapeutic interventions especially for children with autistic spectrum disorder[6, $12,21]$. In the present study, we take a human-centered approach to study gaze in the domain of CHI. Particularly, we focus on the ability of children to recognize and respond to the gaze cues of a robot playing the role of a tutor during a rule gameplay session. Our overall goal is to gain understanding of the child-robot gaze mechanisms to inform the robot's behavior design as a facilitator of children's problem-solving competencies.

\section{METHODOLOGY}

To examine the effects of gaze hints in the context of the educational gameplay with children, we used a matching card game 'Memory.' The study employed a withinsubjects design in which each child interacted with the robot in two conditions: Help (the robot tutor gives gaze clues to help the child find matching cards) and No_Help (the robot tutor only looks at the participant). The aim was to examine whether hints arising from gaze (robot) can improve the performance on a task, measured by time and number of attempts (tries) of Robot Help vs. Robot No_Help. We hypothesized that children would perform better with gaze hints from the tutor than without gaze hints.

\section{Participants}

Eighteen typically developing children, aged between 4 and 11 years, took part in the study; 10 of them were boys and the other 8 were girls. Each child interacted with the robot tutor in two sessions: one under the Help condition and the other under No_Help condition. The whole task took approximately 25 minutes. The experimenter was present at each session to control the robot; they did not initiate any interaction but offered help when requested by 
participants.A picture of the child interacting with NAO can be seen in the figure below:-

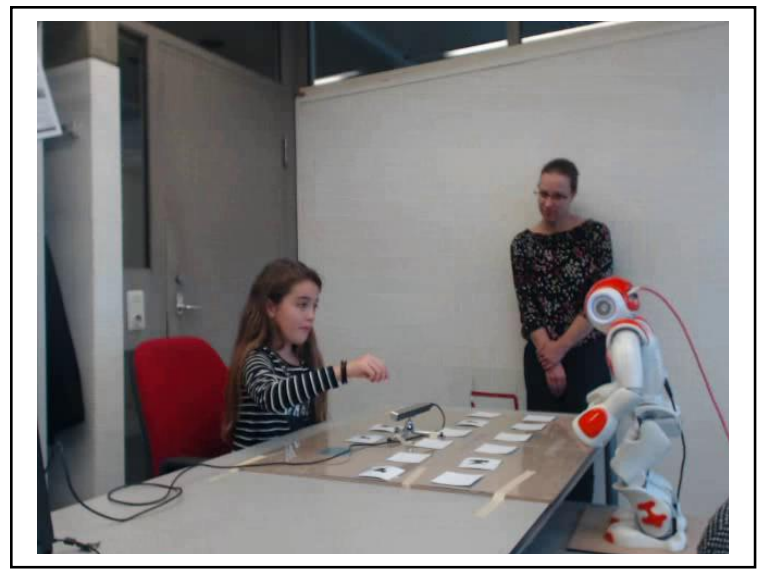

Figure 1. NAO is helping a child to find matching cards; child following the gaze of the NAO

\section{Experiment Setup}

The experimental setup, included a NAO robot, memory game, a webcam, and a personal computer (see Figure 1). $\mathrm{NAO}$ is a $57 \mathrm{~cm}$ tall robot from Aldebaran robotics [33], with a moveable head and facial features that bear resemblance to those of a child. As a result of its minimalistic design and perception capabilities, NAO robot has been adopted widely for research focused on therapeutic training, or for general educational/pedagogical purposes. NAO played the role of the tutor in each of the two sessions. The robot was placed in a fixed position throughout the experiment across the table, approximately $160 \mathrm{~cm}$ from the child.

For the board game design, there were 14 cards arranged in a rectangular layout on the table. The layout had six columns and three rows for a total of 18 cards, or 9 pairs. The distance between the cards on the $\mathrm{X}$-axis was $6 \mathrm{~cm}$; on the $\mathrm{Y}$ - axis, it was $10 \mathrm{~cm}$. In our previous study, we conducted a pilot study to determine whether people can accurately perceive gaze direction and the resolution needed for the head angles of the robot to direct attention at different card locations on the board [28]. Based on our pilot findings, we placed the cards in the first two rows and two cards in the middle positions of the third row, as shown below:-

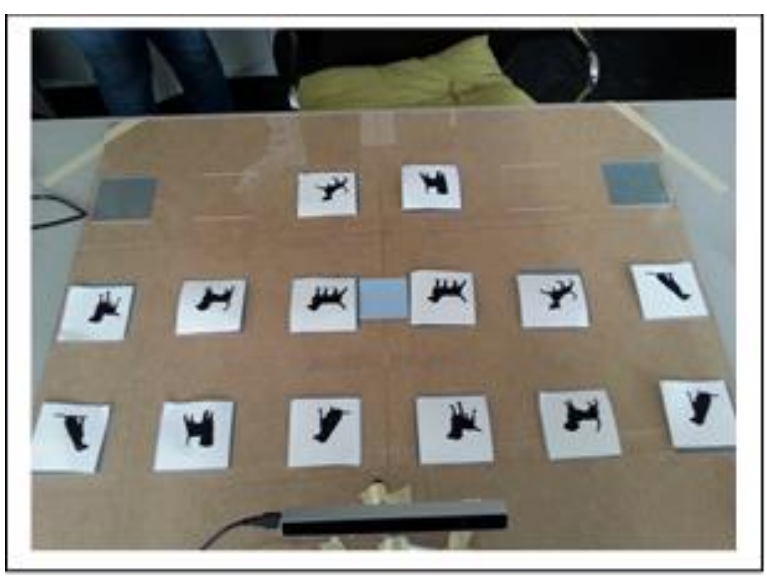

Figure 2. Card arrangement on the board layout.

To develop the memory game algorithm, we used the Java programming language. Each card was labeled with a unique card code and placed in a fixed position on the board layout marked with a head pitch and yaw angle on the computer design. The algorithm was applied such that, after scanning the code of the selected card, the robot automatically executed a sequence of head movements, as follows; the head angles shifted to the position of the chosen card then to the face of the participant, and then to the location of the matching card. The design of the help state gaze for the robot follows the concept of attentiondirecting gaze movement in human communication. Another aspect we considered is the timing of gaze behaviour; we invited fellow students to the lab and played the robot gaze motions to different cards on the table for them with two different timings. We used the timing of head movements that they regarded as more natural. A picture depicting the sequence of NAO help gaze cue can be seen in Figure 3.

\section{Experiment Procedure}

Children were asked to find matching pairs of cards on the table. The cards used for this game contained pictures of black dog 'silhouettes' that varied slightly in shape. In the beginning, the cards were laid face down on the board, and the child was required to find matching pairs of cards by turning the first one and then guess of the matching one. If the two cards turned face up were identical (a pair), the child continued to a new try. Otherwise, the child turned the cards face down and made a new try/move. The game ended when the child found all the matching pairs. The goal of the game was to get all the cards flipped face up (i.e., find all the matching card pairs) in the least number of tries and in the shortest time possible. A 'try' is every time a child flipped over two cards.

We conducted the experiment in the social robotics lab at the university. Before the game session with the robot, the experimenter introduced the child to the robot and detailed the task they were expected to perform. The experimenter 
told the child they were going to play a memory game task in two sessions and the NAO robot would be the tutor. The experimenter asked the child to listen to the instructions from the robot tutor. The robot greeted the child, welcomed them to the session, and gave clear rules of the game saying:

"Hello, welcome to the game session. My name is MACA. I am your tutor. I have a task for you; You are going to find pairs of matching cards on the table. You flip the first card, then flip the second card. If they do not match, turn both back and start over. If they match, leave them turned up. Please go ahead and play the game!"

In the Help condition, the robot tutor added the following statement while giving the instructions" "I know the positions of all the cards on the table; I am going to help you. " However, the tutor did not disclose the modality they were going to use to help; we wanted to see if this statement would trigger a search for help clues from the robot and if children would notice the robot was using gaze to help them. In the No_Help condition, the robot gave the rules of the game and only observed the child during the game.

Each participant interacted with the tutor in both conditions, and we counterbalanced the order of conditions across trials. When the child completed a session, they left the room to allow the experimenter to re-arrange the game and were later welcomed back (approximately after four minutes) for the next session. At the end of each session, the robot verbally thanked the child as follows: "Thank you for playing the game!".

During the experiment, both the experimenter and the researcher were present in the room in each session. The researcher's role was to control the robot and the experimenter's role was to welcome the child, ensure the child was comfortable and offer help when children asked questions or appeared confused. The facilitator sat in a corner in front of a big screen and remained silent for the entire duration and tracked and recorded the actions of the child during the game from the webcam display. After both sessions, the experimenter asked the children a few postexperiment questions on whether they noticed the help cues from the tutor and, if they did, whether that influenced their choice of cards. We also asked what cues they expected the robot tutor would use to help them in the game.

\section{Measurements}

To evaluate the effects of gaze hints on children's behavior, we defined two measures: performance measures and subjective measures as described below:-

Performance Measures: We identified two primary objective measures that are notably used to measure performance in memory game: (1)Duration: the time it takes participants to find all pairs of matching cards on the table; and (2) the number of tries required to find all matching cards. A try consists of choosing two cards. All sessions were video-recorded to facilitate the analysis of these measures.

Subjective Measures: After both sessions, the experimenter asked the children a few post-experiment questions related to the task difficulty, perception of the robot tutor help behavior, and their expectations regarding the robot behavior. During each session, we documented all the observations noted. We also recorded videos to help analyze child-robot interaction regarding child's gaze behavior during the game; the level of engagement during the game and the emotions experienced by children during the game.

\section{RESULTS}

For this analysis, we considered a total of 15 children (age 6 -11; Mean Age= 7.6), for a total of 15 trials in the Help condition and 15 trials in the No_Help conditions. We excluded three (3) children from this analysis, one of the children declined to participate in the second session and the other two were very young (below age 5) and needed a lot of help from the experimenter to play the game.

From the post-experiment interview, eight (8; approximately $53 \%$ ) out of the fifteen (15) children said they noticed the help hints from the tutor, while the others (7: 47\%) stated that they did not see the help gaze cues. Table 1 gives details regarding the children gender, age, and their answers to whether they noticed the help from the tutor (Either YES or NO).

\begin{tabular}{|l|r|r|r|}
\hline Subject & Age & Gender & Noticed_gaze \\
\hline 1 & 9 & Male & YES \\
\hline 2 & 6 & Female & NO \\
\hline 3 & 8 & Male & NO \\
\hline 4 & 6 & Female & NO \\
\hline 5 & 7 & Male & YES \\
\hline 6 & 9 & Female & YES \\
\hline 7 & 6 & Male & NO \\
\hline 8 & 6 & Female & YES \\
\hline 9 & 11 & Female & YES \\
\hline 10 & 9 & Male & YES \\
\hline 11 & 9 & Male & NO \\
\hline 12 & 9 & Female & YES \\
\hline 13 & 6 & Male & NO \\
\hline 14 & 6 & Female & YES \\
\hline 15 & 7 & Female & NO \\
\hline
\end{tabular}

Table 1. Participants details and noticing gaze. 

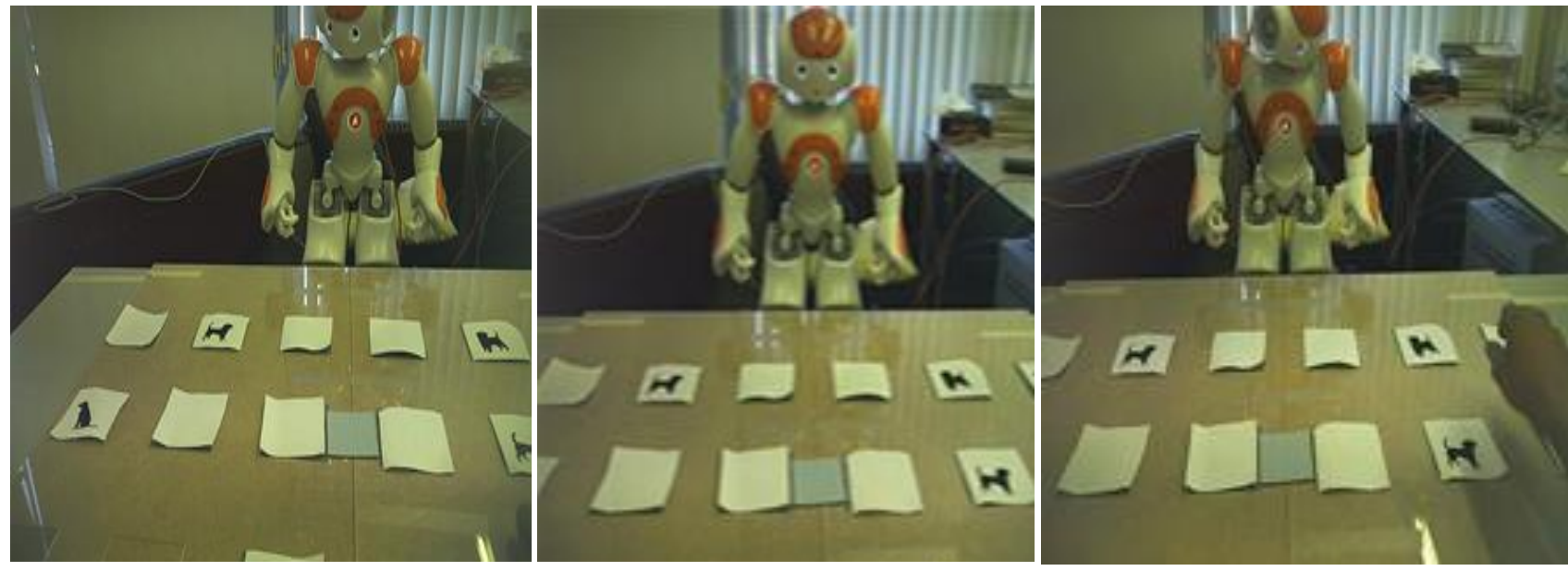

Figure 3. Sequence of help - gaze cue for NAO. NAO Tutor looks at the card picked by the participant, moves its head to the face of the person, and then to the matching card.

\section{Performance Measures}

Duration: We obtained the duration from video recordings, this being the period between the child starting to play the game and completing it in both Help and No_Help conditions.

Number of tries: We counted the number of tries the child used to find matching cards in both Help and No_Help conditions from our video recordings.

\begin{tabular}{|l|r|r|r|}
\hline & \multicolumn{1}{|l|}{ Mean } & \multicolumn{1}{l|}{ SD } & \multicolumn{1}{l|}{ N } \\
\hline Help_Duration & 167.87 & 75.702 & 15 \\
\hline No_Help Duration & 169.6 & 79.18 & 15 \\
\hline Help_NumOfTries & 14.07 & 4.906 & 15 \\
\hline NoHelp_NumOfTries & 17 & 3.645 & 15 \\
\hline
\end{tabular}

Table 2: Descriptive Statistics: Mean Duration and Number of tries

Effect of Help_Type on performance To evaluate the effect of Help on performance, we conducted a repeated measures ANOVA, with Help_Type (Help vs. No_Help) as the within_subject factor.

Duration:- Averages show that participants' duration was slightly higher in the No_Help condition than in the Help condition $($ Help $=167.87 \mathrm{Sec}$; No_Help $=169.6 \mathrm{Sec}$ ). However, we found no significant difference in duration between the Help and No_Help conditions (F $(1,14)$ $=0.015, \mathrm{p}=0.905$ ). Furthermore, there was no significant correlation between noticing gaze and the duration the children took to play the game ( $\mathrm{p}=0.530$, two-tailed), thus there was no significant difference in time taken to find all the matching pairs between those who noticed gaze hints and those who did not notice the gaze hints.
Number of tries:- Also, participants used fewer tries when there was Help in the game than when there was no help provided by the tutor $($ Help $=14.07$ tries; No_Help $=17$ tries). Univariate tests on the effects of Help_Type show a significant main effect on the number of tries $(\mathrm{F}(1,14)$ $=5.331, p=0.037)$.). Further analysis shows a significant correlation between noticing gaze hints and the number of tries $(\mathrm{p}=0.001$, two-tailed). This shows that the children who reported seeing the gaze hints in the robot behavior performed significantly better, measured by the number of tries, than those who did not identify the gaze cues.

Age \& Gender: - We found no correlation between gender and noticing help ( $\mathrm{p}=0.483$, two-tailed). However, in our observation, we noticed age had an influence on the capacity of children to read help from gaze with older children being more aware of the tutor intent and behaving accordingly while most younger children were not able to interpret the robot was helping them despite noticing the cues. This can be explained by social cognition developmental theories of children including the theory of mind [7].

\section{DISCUSSION \& CONCLUSION}

In this study, we examined whether robot gaze hints can improve the performance of children in a matching card game. We compared two performance measures (time and number of tries) in two conditions, one in which the robot provided gaze hints to help the child find matching cards, and one in which the robot did not provide help to the children. We analyzed the data of 15 children, who were between the ages of 6 and 11. In the following paragraphs, we discuss our performance measures results based on our observations during the study and the answers from children in the post-experiment interview.

In our first hypothesis, we projected that children would perform better with help (gaze hints) from the tutor than 

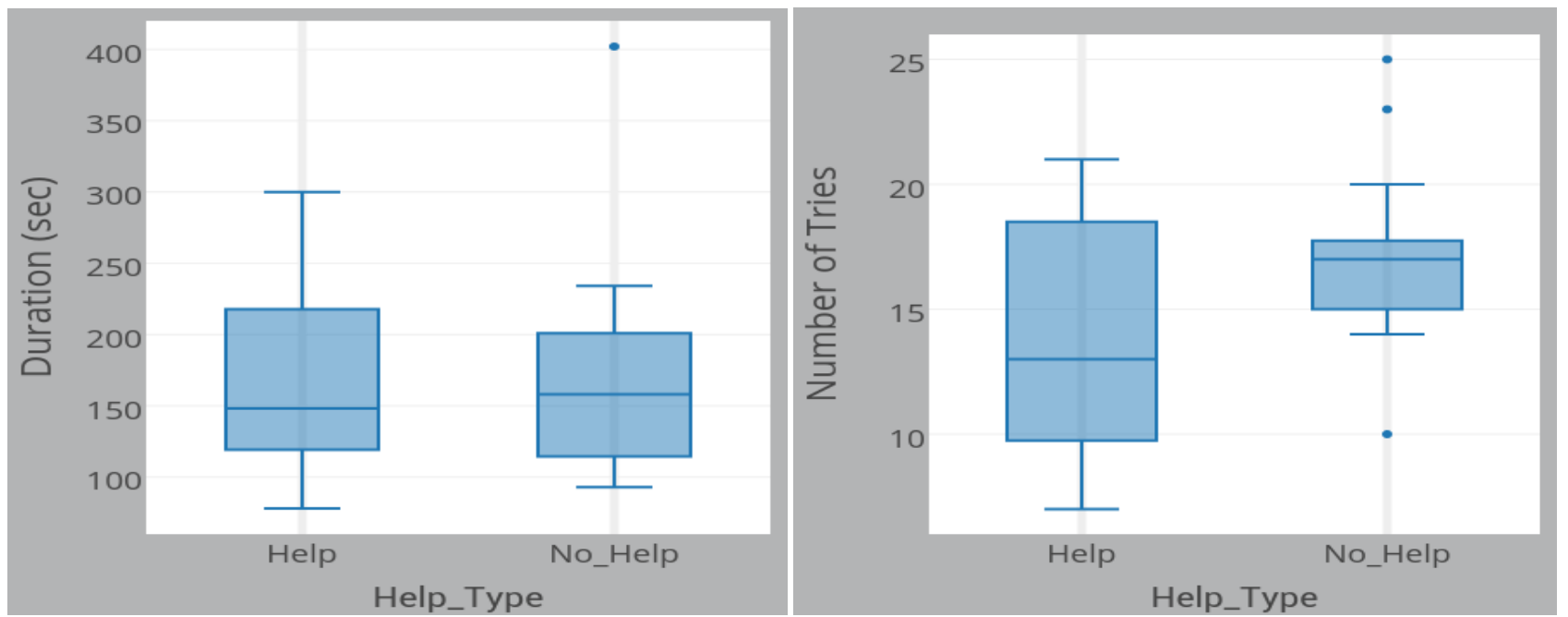

Figure 4. (Left) The duration in (seconds) to find all matching cards with and without help. (Right) The number of tries to find all matching cards with and without help.

without help. Analysis of the number of tries supported this assumption. We found overall that children performed better with gaze hints from the tutor than without gaze hints. However, we found no significant time difference between the two conditions of Help and No_Help.

8 out of the 15 children said they noticed the robot hints during the game, while the other children stated that they did not notice the help hints. The majority of the children who did not notice the gaze hints said they noted the head movements of the robot tutor but did not understand that the robot was using gaze to help them, so they were unable to interpret the head movements as gestures pointing to the matching card.

We further found that children who noticed the help gaze hints from the robot performed significantly better with significantly fewer attempts than those who did not see the help. However, we found no difference in duration between those who noticed the gaze hints and those who did not. Thus, based on the findings, we can conclude that most of the children who noticed the gaze hints were able to interpret them and attribute meaning to accept them as help cues. From the subjective feedback we got from the postexperiment interview, children who noticed the help from the robot regarded the robot as friendly giving it terms such as "cool" and were engaged longer during the interaction with the robot.

We found no significant difference in durations during the No_help and Help condition. Moreover, there was no significant correlation between noticing gaze and the duration. There are several possible explanations for this. Firstly, as soon as the children noticed that the robot tutor was helping them with gaze hints, they waited until the robot showed them the matching card, even when they had an idea of where the matching card was. Secondly, the novelty effect of the robot, which is supported by the larger duration for the robot even when the number of tries was less, indicates that the children who noticed gaze probably spent more time looking at the robot. Another probable reason is the duration of head motions during attention shifts from the flipped card to the face of the participant and then to the matching card. Lastly, a few of the children spent some time asking the experimenter questions during the game either due to confusion, or when they saw the robot movement and could not interpret what it was doing.

The results from this study compare with our previous study with adult participants using a similar setting on performance. In the adult setting, we found that the participants performed better when the tutor was helping than without help [29]. However, from our observations, we can highlight notable differences on how children perceive and interpret gaze cues from the robot. For example, in the child experiment, we observed a few children who proceeded to select different cards despite noticing that the robot tutor was looking at a particular card, which was not the case for the adult participants. This could be attributed to social cognitive theories of development $[7,24]$, which are fascinating to study with robots. This could be attributed to social cognitive theories of development -such as perspective taking and agency attribution- which are fascinating to study with robots. This work provides initial findings on the ability of children to read, perceive and attribute intentions to a robot gaze.

Also, in both settings for the adult and children, most indicated that they expected verbal help from the tutor. We assume the design of the robot behavior may have led participants to expect verbal help from the tutor since, in the beginning, the robot verbally gave instructions to the participant but remained silent during the entire interaction only giving gaze clues. In future works, it would be interesting to vary levels of nonverbal cues -such as a quick 
glance-, and introducing the fairness of the tutor as a study variable on performance and subjective experience.

Currently, we are carrying out an observational analysis of the child-robot interaction videos recorded to examine child-robot dyad gaze behavior during the game (such as patterns of joint attention and eye contact), the level of engagement and the emotions expressed throughout the game - flow. Our future work involves as well examining the temporal aspects of gaze in human-human interactions to build more realistic interactive robot gaze behaviors, and study interactive gaze behavior with robots with articulated eyes.

\section{ACKNOWLEDGMENTS}

\section{REFERENCES}

1. Admoni, H., Hayes, B., Feil-Seifer, D., Ullman, D., \& Scassellati, B. (2013, March). Are you looking at me? perception of robot attention is mediated by gaze type and group size. In Proceedings of the 8th

ACM/IEEE international conference on human-robot interaction (pp. 389-396).

2. Admoni, H. \& Scassellati, B., 2017. Social Eye Gaze in Human-Robot Interaction: A Review. , 6(1), pp.25-63.

3. Andrist, S., Tan, X. Z., Gleicher, M., \& Mutlu, B. (2014, March). Conversational gaze aversion for humanlike robots. In Proceedings of the 2014 ACM/IEEE international conference on Human-robot interaction (pp. 25-32)

4. Argyle, M., Ingham, R., Alkema, F., \& McCallin, M. (1973). The different functions of gaze. Semiotica, 7(1), 19-32.

5. Astington, J. W., \& Jenkins, J. M. (1995). Theory of mind development and social understanding. Cognition \& Emotion, 9(2-3), 151165.

6. Barakova, E. I., Bajracharya, P., Willemsen, M., Lourens, T., \& Huskens, B. (2015). Long-term LEGO therapy with humanoid robot for children with ASD. Expert Systems, 32(6), 698-709.

7. Baron-Cohen, S. (1997). Mindblindness: An essay on autism and theory of mind. MIT press.

8. Belpaeme, Tony, Paul E. Baxter, Robin Read, Rachel Wood, Heriberto Cuayáhuitl, Bernd Kiefer, Stefania Racioppa et al. "Multimodal child-robot interaction: Building social bonds." Journal of Human-Robot Interaction 1, no. 2 (2012): 33-53.

9. Boucher, J. D., Pattacini, U., Lelong, A., Bailly, G., Elisei, F., Fagel, S., ... \& Ventre-Dominey, J. (2012). I reach faster when I see you look: gaze effects in human-human and human-robot face-to-face cooperation. Frontiers in neurorobotics, 6, 3.

10. Brooks, R., \& Meltzoff, A. N. (2014). Gaze following: a mechanism for building social connections between infants and adults. In Mechanisms of social connection: from brain to group, 167-183.

11. Broz, F., Lehmann, H., Nakano, Y., \& Mutlu, B. (2012, March). Gaze in HRI: from modeling to communication. In Proceedings of the seventh annual ACM/IEEE international conference on HumanRobot Interaction (pp. 491-492).

12. Dautenhahn, K. (2003). Roles and Functions of Robots in Human Society: Implications from Research in Autism Therapy. Robotica, 21(4), 443452.

13. Dominey, P.F., 2017. I Reach Faster When I See You Look: Gaze Effects in Human - Human and Human - Robot Face-to-Face Cooperation I reach faster when I see you look : gaze effects in human - human and human - robot face-to-face cooperation. , (May 2012)

14. Emery, N. J. (2000). The eyes have it: the neuroethology, function and evolution of social gaze. Neuroscience \& Biobehavioral Reviews, 24(6), 581604.

15. Frischen, A., Bayliss, A. P., \& Tipper, S. P. (2007). Gaze cueing of attention: visual attention, social cognition, and individual differences. Psychological bulletin, 133(4), 694.

16. Goren Gordon, Cynthia Breazeal, and Susan Engel. 2015. Can Children Catch Curiosity from a Social Robot?. In Proceedings of the Tenth Annual ACM/IEEE International Conference on HumanRobot Interaction (HRI '15). ACM, New York, NY, USA, 91-98.

17. Huskens, B., Verschuur, R., Gillesen, J., Didden, R., $\&$ Barakova, E. (2013). Promoting question-asking in school-aged children with autism spectrum disorders: Effectiveness of a robot intervention compared to a human-trainer intervention. Developmental neurorehabilitation, 16(5), 345-356.

18. Imai, M., Kanda, T., Ono, T., Ishiguro, H., \& Mase, K. (2002). Robot mediated round table: Analysis of the effect of robot's gaze. In Robot and Human Interactive Communication, 2002. Proceedings. 11th IEEE International Workshop on (pp. 411-416).

19. Kendon, A. (1967). Some functions of gaze-direction in social interaction. Acta psychologica, 26, 22-63.

20. Kleinke, C. L. (1986). Gaze and eye contact: a research review. Psychological bulletin, 100(1), 78. 
21. Kozima, H., Nakagawa, C., \& Yasuda, Y. (2007). Children-robot interaction: a pilot study in autism therapy. Progress in Brain Research, 164, 385-400.

22. Krämer, N. C., Eimler, S., von der Pütten, A., \& Payr, S. (2011). Theory of Companions: What Can Theoretical Models Contribute To Applications and Understanding of Human-Robot Interaction? Applied Artificial Intelligence, 25(6), 474-502.

23. Langton, S.R.H., Watt, R.J. \& Bruce, V., 2000. Do the eyes have it? Cues to the direction of social attention. , 4(2), pp.50-59.

24. Meltzoff, A. N., Brooks, R., Shon, A. P., \& Rao, R. P. (2010). "Social" robots are psychological agents for infants: A test of gaze following. Neural Networks, 23(8), 966-972.

25. Moon, Aj. et al., 2014. Meet me where i'm gazing. Proceedings of the 2014 ACM/IEEE international conference on Human-robot interaction - HRI '14, pp.334-341.

26. Mutlu, B. (2009). Designing gaze behavior for humanlike robots (Doctoral dissertation, Ford Motor Company).

27. Mutlu, B., Yamaoka, F., Kanda, T., Ishiguro, H., \& Hagita, N. (2009, March). Nonverbal leakage in robots: communication of intentions through seemingly unintentional behavior. In Proceedings of the 4th ACM/IEEE international conference on Human robot interaction (pp. 69-76).

28. Anonymized.

29. Anonymized.

30. Palinko, O., Sciutti, A., Schillingmann, L., Rea, F., Nagai, Y., \& Sandini, G. (2015, August). Gaze contingency in turn-taking for human robot interaction: Advantages and drawbacks. In Robot and Human Interactive Communication (RO-MAN), 2015 24th IEEE International Symposium on (pp. 369374).

31. Pfeiffer-Lessmann, N., Pfeiffer, T., \& Wachsmuth, I. (2012). An operational model of joint attentiontiming of gaze patterns in interactions between humans and a virtual human. In Proceedings of the 34th Annual Conference of the Cognitive Science Society (CogSci 2012), N. Miyake, D. Peebles, and RP Cooper, Eds. Austin, TX USA: Cognitive Science Society (pp. 851-856).

32. Poggi, I., Pelachaud, C., \& De Rosis, F. (2000). Eye communication in a conversational 3D synthetic agent. AI communications, 13(3), 169-181. Palinko, O. et al., Gaze Contingency in Turn - Taking for Human Robot Interaction: Advantages and Drawbacks.
33. Robotics, A. (2010). Nao robot reference manual. Version 1.10. 10.

34. Ruhland, K., Andrist, S., Badler, J., Peters, C., Badler, N., Gleicher, M., ... \& Mcdonnell, R. (2014, April). Look me in the eyes: A survey of eye and gaze animation for virtual agents and artificial systems. In Eurographics State-of-the-Art Report (pp. 69-91).

35. Yamazaki, A., Yamazaki, K., Kuno, Y., Burdelski, M., Kawashima, M., \& Kuzuoka, H. (2008, April). Precision timing in human-robot interaction: coordination of head movement and utterance. In Proceedings of the SIGCHI C onference on Human Factors in Computing Systems (pp. 131-140).

36. Yoshikawa, Y., Shinozawa, K., Ishiguro, H., Hagita, N., \& Miyamoto, T. (2006, August). Responsive Robot Gaze to Interaction Partner. In Robotics: Science and systems

37. Yu, C., Schermerhorn, P., \& Scheutz, M. (2012). Adaptive eye gaze patterns in interactions with human and artificial agents. ACM Transactions on Interactive Intelligent Systems (TiiS), 1(2), 13.

38. Wiese E, Müller HJ, Wykowska A: Using a GazeCueing Paradigm to Examine Social Cognitive Admoni, H. \& Scassellati, B., 2017. Social Eye Gaze in Human-Robot Interaction: A Review. , 6(1), pp.25-63. 